\title{
Reporting guidelines for human microbiome research: the STORMS checklist
}

Chloe Mirzayi ${ }^{1}$, Audrey Renson ${ }^{2}$, Genomic Standards Consortium, Massive Analysis and Quality

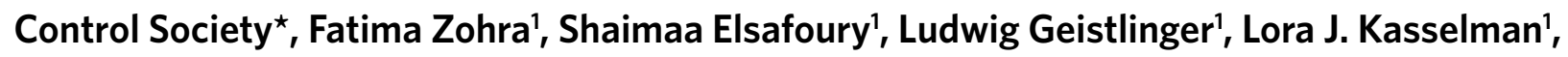
Kelly Eckenrode', Janneke van de Wijgert ${ }^{3}$, Amy Loughman ${ }^{4}$, Francine Z. Marques ${ }^{5}$, David A. Maclntyre ${ }^{6}$, Manimozhiyan Arumugam ${ }^{7}$, Rimsha Azhar ${ }^{1}$, Francesco Beghini ${ }^{8}$, Kirk Bergstrom ${ }^{9}$, Ami Bhatt ${ }^{10}$, Jordan E. Bisanz ${ }^{11}$, Jonathan Braun ${ }^{12}$, Hector Corrada Bravo ${ }^{13}$, Gregory A. Buck ${ }^{14}$, Frederic Bushman ${ }^{15}$, David Casero ${ }^{16}$, Gerard Clarke ${ }^{17}$, Maria Carmen Collado ${ }^{18}$, Paul D. Cotter ${ }^{19,20,21}$, John F. Cryan ${ }^{20,22}$, Ryan T. Demmer ${ }^{23}$, Suzanne Devkota ${ }^{16}$, Eran Elinav ${ }^{24,25}$, Juan S. Escobar ${ }^{26}$, Jennifer Fettweis ${ }^{14}$, Robert D. Finn ${ }^{27}$, Anthony A. Fodor ${ }^{28}$, Sofia Forslund ${ }^{29}$, Andre Franke ${ }^{30}$, Cesare Furlanello ${ }^{31}$, Jack Gilbert ${ }^{32}$, Elizabeth Grice ${ }^{33}$, Benjamin Haibe-Kains ${ }^{34}$, Scott Handley ${ }^{35}$, Pamela Herd ${ }^{36}$, Susan Holmes ${ }^{37}$, Jonathan P. Jacobs ${ }^{38}$, Lisa Karstens ${ }^{39}$, Rob Knight ${ }^{40}$, Dan Knights ${ }^{41,42}$, Omry Koren ${ }^{43}$, Douglas S. Kwon ${ }^{44}$, Morgan Langille ${ }^{45}$, Brianna Lindsay ${ }^{46}$,

Dermot McGovern ${ }^{16}$, Alice C. McHardy ${ }^{47}$, Shannon McWeeney ${ }^{48}$, Noel T. Mueller ${ }^{49}$, Luigi Nezi ${ }^{50}$, Matthew Olm ${ }^{51}$, Noah Palm ${ }^{52}$, Edoardo Pasolli53, Jeroen Raes ${ }^{54}$, Matthew R. Redinbo ${ }^{55}$, Malte Rühlemann ${ }^{56}$, R. Balfour Sartor ${ }^{57}$, Patrick D. Schloss ${ }^{58}$, Lynn Schriml ${ }^{59}$, Eran Segal ${ }^{60}$, Michelle Shardell ${ }^{59}$, Thomas Sharpton ${ }^{61}$, Ekaterina Smirnova ${ }^{62}$, Harry Sokol ${ }^{63}$, Justin L. Sonnenburg ${ }^{51}$, Sujatha Srinivasan ${ }^{64}$, Louise B. Thingholm ${ }^{56}$, Peter J. Turnbaugh ${ }^{65}$, Vaibhav Upadhyay ${ }^{65}$, Ramona L. Walls ${ }^{66}$, Paul Wilmes ${ }^{67,68}$, Takuji Yamada ${ }^{69}$, Georg Zeller ${ }^{70}$, Mingyu Zhang ${ }^{49}$, Ni Zhao ${ }^{71}$, Liping Zhao ${ }^{72}$, Wenjun Bao ${ }^{73}$, Aedin Culhane ${ }^{74}$, Viswanath Devanarayan ${ }^{75}$, Joaquin Dopazo ${ }^{76}$, Xiaohui Fan ${ }^{77}$, Matthias Fischer ${ }^{78,79}$, Wendell Jones ${ }^{80}$, Rebecca Kusko ${ }^{81}$, Christopher E. Mason ${ }^{82}$, Tim R. Mercer ${ }^{83}$, Susanna-Assunta Sansone ${ }^{84}$, Andreas Scherer ${ }^{85}$, Leming Shi ${ }^{86}$, Shraddha Thakkar ${ }^{87}$, Weida Tong ${ }^{88}$, Russ Wolfinger ${ }^{89}$, Christopher Hunter ${ }^{90}$, Nicola Segata ${ }^{8,50}$, Curtis Huttenhower (1) 91,93 , Jennifer B. Dowd ${ }^{92,93}$, Heidi E. Jones ${ }^{1,93}$ and Levi Waldron $(1,93 \bowtie$

The particularly interdisciplinary nature of human microbiome research makes the organization and reporting of results spanning epidemiology, biology, bioinformatics, translational medicine and statistics a challenge. Commonly used reporting guidelines for observational or genetic epidemiology studies lack key features specific to microbiome studies. Therefore, a multidisciplinary group of microbiome epidemiology researchers adapted guidelines for observational and genetic studies to culture-independent human microbiome studies, and also developed new reporting elements for laboratory, bioinformatics and statistical analyses tailored to microbiome studies. The resulting tool, called 'Strengthening The Organization and Reporting of Microbiome Studies' (STORMS), is composed of a 17-item checklist organized into six sections that correspond to the typical sections of a scientific publication, presented as an editable table for inclusion in supplementary materials. The STORMS checklist provides guidance for concise and complete reporting of microbiome studies that will facilitate manuscript preparation, peer review, and reader comprehension of publications and comparative analysis of published results.

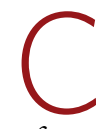

hanges in the human microbiome have been associated with many disease and health states ${ }^{1}$. However, reporting the results of human microbiome research is challenging, as it often involves approaches from microbiology, genomics, biomedicine, bioinformatics, statistics, epidemiology and other fields, which results in a lack of consistent recommendations for the reporting of methods and results. Inconsistent reporting can have consequences for the field by affecting the reproducibility of study results ${ }^{2}$. Although researchers have called for better reporting standards ${ }^{3}$, such as the Genomic Standards Consortium's MIxS checklist ${ }^{4}$, to 
provide a means for reporting sampling, processing and data generation, no comprehensive standardized guidelines spanning laboratory and epidemiological reporting of microbiome studies have been proposed.

Standard reporting guidelines promote research consistency and, as a consequence, encourage reproducibility and improved study design. Editorial adoption of the CONSORT (Consolidated Standards of Reporting Trials) guidelines, for example, has been associated with an increase in the quality of trial reporting ${ }^{5,6}$. Other epidemiological reporting guidelines have seen broad adoption, such as STROBE (Strengthening the Reporting of Observational studies in Epidemiology) ${ }^{7}$ and STREGA (Strengthening the Reporting of Genetic Association Studies) ${ }^{8}$. STROBE-metagenomics ${ }^{9}$ proposes an extension to the STROBE checklist for metagenomics studies. Subsequent to the MIAME (Minimum Information About a Microarray Experiment) checklist ${ }^{9}$, the MIMARKS (Minimum Information about a MARKer gene Sequence) and MIxS (Minimum Information about any (x) Sequence) checklists provide detailed guidance on the reporting of sequencing studies in general. These are focused on the technical aspects of data generation, however, as are projects such as the MBQC (Microbiome Quality Control) project $^{10}$ and IHMS (International Human Microbiome Standards) ${ }^{11,12}$. Together, these serve as useful foundations, but they do not span the full range of reporting of human microbiome studies or include items intended for other types of studies, and they provide limited guidance on manuscript preparation.

Studies of the human microbiome share many features with studies of other types of molecular epidemiology, but they also require unique considerations with their own methodological best practices and reporting standards. In addition to standard elements of epidemiological study design, culture-independent microbiome studies involve the collection, handling and preservation of biological specimens; evolving approaches to laboratory processing with elevated potential for batch effects; bioinformatics processing; statistical analysis of sparse, unusually distributed, high-dimensional data; and reporting of results on potentially thousands of microbial features ${ }^{13-15}$. Because there is no agreed-upon gold-standard method for microbiome research and the field has not reached consensus on many of these aspects, inconsistencies in reporting inhibit reproducibility and hamper efforts to draw conclusions across similar studies.

For these reasons, we convened a multi-disciplinary working group to develop guidelines tailored to microbiome study reporting. Members of this group include epidemiologists, biostatisticians, bioinformaticians, physician-scientists, genomicists and microbiologists. The checklist is designed to balance completeness with burden of use and is applicable to a broad range of human microbiome study designs and analysis. The STORMS (Strengthening The Organizing and Reporting of Microbiome Studies) checklist (Supplementary Table 1) draws relevant items from related guidelines and adds new tailored guidelines to serve as a tool to organize study planning and manuscript preparation, to improve the clarity of manuscripts and to facilitate reviewers and readers in assessing these studies.

\section{Methodology}

STORMS was the result of a collaborative development process. In this section we discuss the methodology used to prepare the STORMS guidelines.

Origin and development. The origins of these guidelines are rooted in a project to create a standardized database of published literature reporting relationships between the microbiome and disease (https://bugsigdb.org/; website in preparation). The goal of that project is to create a publicly available, standardized database of microbiome study findings indexed by condition of interest (for example, disease, health status, diet or environmental factor), microbiome site (for example, gut, mouth or skin) and microbial taxonomy to aid comparative analysis. As of August 2021, 31 curators (Supplementary Table 2) had extracted findings from 513 unique published studies (Supplementary Table 3). Included studies must have examined the relationship between the microbiome and a condition of interest and must have included findings on a taxonomic level (even if all findings were null).

This review revealed substantial reporting heterogeneity, particularly for epidemiology, such as study design, confounding factors and sources of bias. It also revealed microbiome-specific issues, including statistical analysis of compositional relative abundance data and handling of 'batch' effects ${ }^{16}$. This heterogeneity highlighted the need for standardized reporting guidelines, similar to those used in other fields of study. The curators determined that standardized reporting guidelines would streamline the review process but would also, more importantly, help researchers throughout the field of microbiome research communicate their findings effectively.

The resulting multidisciplinary group of bioinformaticians, epidemiologists, biostatisticians and microbiologists was thus convened to discuss microbiome reporting standards. The group began by reviewing existing reporting standards, including STROBE $^{17}$, STROBE-ME $^{18}$, STREGA $^{8}, \mathrm{MICRO}^{17}, \mathrm{MIMARKS}^{4}$ and STROGAR ${ }^{19}$. The group also reviewed existing articles containing recommendations for microbiome reporting ${ }^{20,21}$. The STROBE and STREGA guidelines were used as a starting point for the STORMS checklist, although aspects were incorporated from the other reporting standards.

Following the guidelines on the development of reporting standards recommended by EQUATOR, the group created a comprehensive list of potential guideline items. From this list, group members added, modified and removed items on the basis of their expertise. After the first round of edits, the checklist was then applied to a recent microbiome study ${ }^{22}$ by group members. Comments, removals and additions were harmonized after each round. On the basis of this process, additional changes, simplifications and clarifications were made. This process was repeated until there was a group consensus that the checklist was ready for use.

In addition to the core working group, outside subject-matter experts identified by members of the working group were then invited to review the guidelines and provide feedback as members of the STORMS Consortium. Substantive feedback (that is, not grammar, spelling or other small changes) from 46 authors was organized by topic and compiled into a feedback document, and this was responded to as in a response to reviewer's letter. After this round of revisions, consortium members were once again invited to review the checklist before submission for publication.

\section{Elaboration and explanation of checklist items}

This section describes the items in the STORMS checklist.

Checklist. The latest version of the checklist and a summary of items at the time of publication are presented in Supplementary Table 1; updated versions will be posted online (https://stormsmicrobiome.org). Of the items in the latest version in the STORMS checklist, nine items or sub-items were unchanged from STROBE, three were modified from STROBE, one was modified from STREGA, and fifty-seven new guidelines were developed. Nine items that overlap MIxS are specified. Rationales for new and modified items are presented below. Documentation of items unmodified from STROBE and STREGA were presented in the publications of those checklists.

Abstract (1.0-1.3). Along with commonly included abstract materials, such as a basic description of the participants and results, authors should report the study design ${ }^{23}$ - such as a cross-sectional, 
case-control, cohort or randomized controlled trial - in the abstract of their article (item 1.1), as required by other reporting guidelines. Communication of the study design in the abstract allows readers to quickly categorize the type of evidence provided. As part of this basic description, sequencing methods should be mentioned (item 1.2). Body site(s) sampled should also be included (item 1.3).

Introduction (2.0-2.1). The introduction should clearly describe the underlying background, evidence or theory that motivated the current study (item 2.0). Among other possibilities, this could include pilot study data, previous findings from a similar study or topic or a biologically plausible mechanism that has been proposed. This clarifies for the reader the motivations for the present study. If the study is exploratory in nature, the introduction should explain what motivated the current exploration and the goals of the exploratory study. The hypothesis developed on the basis of the background should be included. If the study was exploratory and did not define a hypothesis, pre-specified study objectives should be included (item 2.1).

Methods (3.0-8.5). Methods constitute a majority of the checklist, as outlined below.

Participants (3.0-3.9). The methods section should contain sufficient information for study replicability. Because study design is essential to understanding a study, this should be stated in the methods (item 3.0). In the description of the participants in the study, the population of interest should be described, as well as how participants were sampled from the source population (item 3.1). Because participant characteristics such as environment ${ }^{24}$, lifestyle behaviors, diet, biomedical interventions, demographics ${ }^{25}$ and geography ${ }^{26}$ (item 3.2) can correspond with substantial differences in the microbiome, it is essential to include this description. Temporal context can be important as well, so start and end dates for recruitment, follow-up and data collection should be stated (item 3.3).

Specific criteria used to assess potential participants for eligibility in the study should also be reported, with details of both inclusion criteria and exclusion criteria (item 3.4). Inclusion and exclusion criteria are pre-established characteristics used for the selection of participants into a study, and describing these criteria is essential for understanding a study's target population ${ }^{27}$. This is expanded from STROBE, which requires eligibility criteria but does not specify that both inclusion criteria and exclusion criteria should be reported in detail. Any information collected about antibiotics or other treatments that could affect the microbiome should be described (item 3.5), as well as if any exclusion criteria included recent use of antibiotics or other medications.

The final analytic sample sizes should be stated, as well as the reason for any exclusion of participants at any step of the recruitment, follow-up or laboratory processes (item 3.6). STROBE suggests using a flowchart to show when and why participants were removed from the study. A template for such flowcharts is presented here (Fig. 1), and a public-domain version is available for re-use online (https://stormsmicrobiome.org/figures). If participants were lost to follow-up or did not complete all assessments in a longitudinal study, details on how follow-ups were conducted should be stated and time-point-specific sample sizes should also be reported (item 3.7). Additionally, studies that matched cases to controls should describe what variables were used in matching (item 3.8).

Laboratory methods (4.0-4.17). Since STROBE does not cover laboratory methods, new items were developed for the STORMS checklist. Laboratory methods should be described in sufficient detail to allow replication. The handling of lab samples should be described, including procedures for sample collection (item 4.1), shipping (item 4.2) and storage (item 4.3).
Because DNA extraction can be a major source of technical differences across studies ${ }^{10}$, DNA extraction methods should be described (item 4.4). Description of the removal of human DNA and enrichment for microbial DNA, if performed, should also be included (item 4.5). Likewise, if positive controls (item 4.7), negative controls (item 4.8) or contaminant-mitigation methods (item 4.9) were used, they should be identified and described.

Sequencing-related methods should be reported. This includes primer selection and DNA amplification (including the variable region of the $16 \mathrm{~S}$ rRNA gene, if applicable) (item 4.6). Major divisions of sequencing strategy, such as shotgun or amplicon sequencing, should be identified (item 4.11). Finally, the methods used to determine relative abundances should be explained (item 4.12), and the read numbers that serve as denominators should be recorded.

Batch effects should be discussed as a potential source of confounding, including steps taken to ensure batch effects do not overlap exposures or outcomes of interest (item 4.13) ${ }^{28}$. If metatranscriptomics, metaproteomics or metabolomics are conducted, details of those methods should be provided (items 4.14-4.16).

Data sources/measurement (5.0). For non-microbiome data (for example, health outcomes, participant socioeconomic, behavioral, dietary and biomedical characteristics, including disease location and activity, and environmental variables), the measurement and definition of each variable should be described (item 5.0). For example, a participant's sex and age could be obtained from electronic medical records or from a questionnaire distributed to participants; this data source should be described. Limitations of measurements may also be discussed, including potential bias due to misclassification or missing data, as well as any attempts made to address these measurement issues.

Research design considerations for causal inference (6.0-6.1). Observational data are often used to test associations that aim toward causal inference in situations in which the hypothesized causal relationship is not directly observed. Methods include, for example, the use of multivariable analysis or matching to adjust for confounding variables between a hypothesized exposure (such as abundance of a microbial taxon) and the disease or condition under study $^{29}$. Confounders can be thought of as common causes of the exposure and the outcome under study that can induce a spurious association between the exposure and the outcome $e^{30,31}$. For example, age could be a common confounder due to its influence on the microbiome and on the risk of most health outcomes ${ }^{32}$. Laboratory batch effects could also confound relationships between the microbiome and a condition of interest if steps are not taken to avoid imbalance of the condition across batches ${ }^{33}$. A common method for attempting to control for measured confounding is to adjust for or stratify on the confounder ${ }^{30}$. Justification should be provided for variables included or excluded in regression models for causal inference (item 6.0), as adjusting for or stratifying on a non-confounding variable can introduce bias ${ }^{34}$. As part of this theoretical justification, authors should consider including a directed acyclic graph showing the hypothesized causal relationships of interest ${ }^{35,36}$.

In addition to consideration of the theoretical motivations for the present study, the potential for selection or survival bias that can distort the observed relationship between the microbiome and variable of interest should be discussed (item 6.1). For example, such bias may occur due to loss to follow-up (in longitudinal studies) or due to lack of inclusion of participants in the study due to the condition itself (for example, participants who have died of aggressive forms of colorectal cancer and have not survived to be in a hypothetical study of colorectal cancer microbiomes) ${ }^{37}$. Other items elsewhere in the checklist may be directly relevant to questions of causal inference, including hypotheses (item 2.1), study design (item 3.0), matching (item 3.8), bias (item 13.1) and generalizability 
STORMS analytic sample size flowcharts (item 3.6)

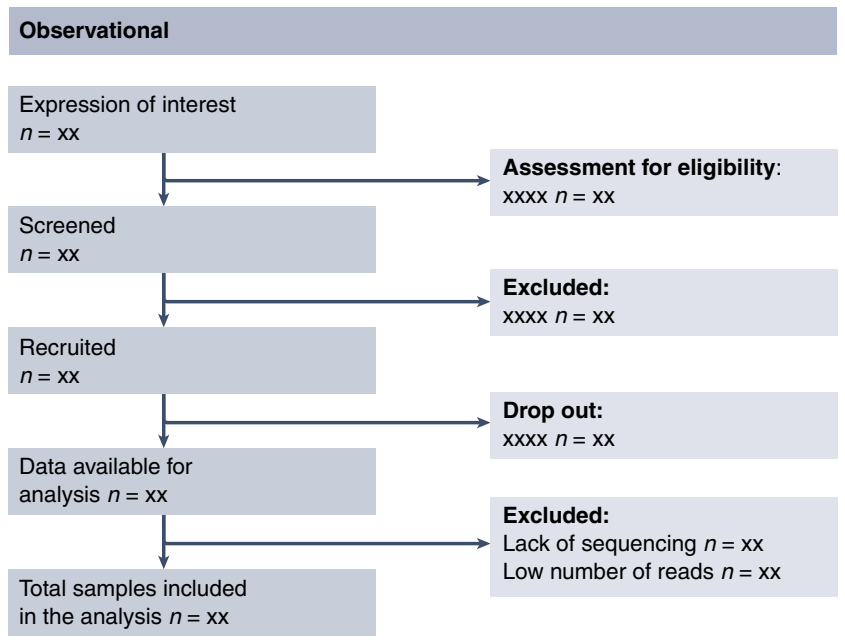

Experimental

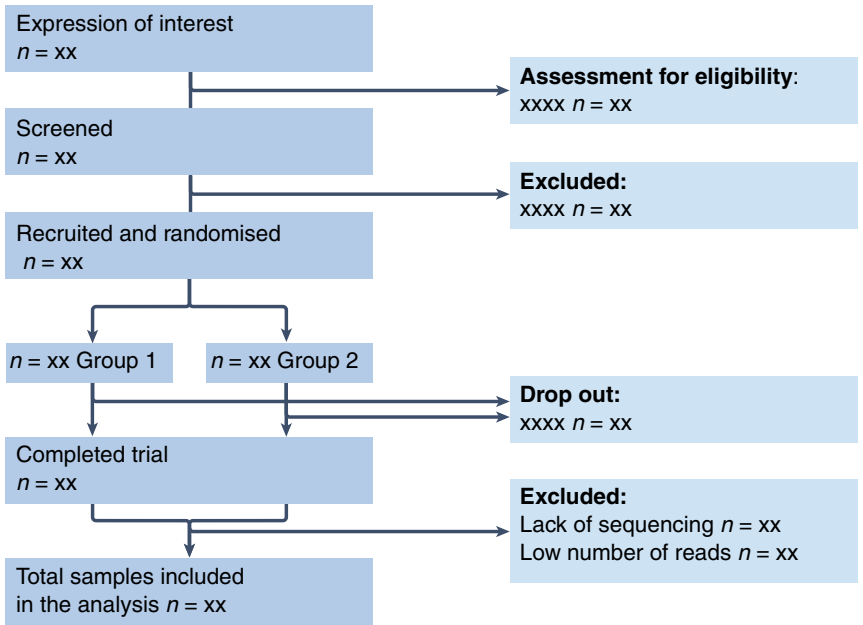

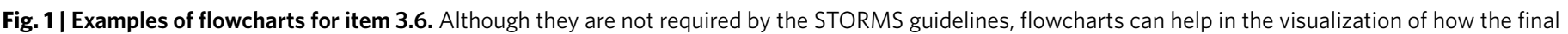
analytic sample was calculated.

(item 13.2). Authors investigating causal questions are encouraged to consider their reporting on these items in the context of causal inference as well.

Bioinformatics and Statistical Methods (7.0-7.9). Adequate description of bioinformatics and statistical methods is essential to the production of a rigorous and reproducible research report. Data transformations (such as normalization, rarefaction and percentages) should be described (item 7.0). Quality-control methods should be fully disclosed, including criteria for filtering or removing reads or samples (item 7.1). All statistical methods used to analyze the data should be stated (item 7.3), including how results of interest were selected (for example, use of a $P$ value, $q$ value or other threshold) (item 7.8). Taxonomic, functional profiling or other sequence analysis methods should be described in detail (item 7.2). In the interest of reproducibility, all software, packages, databases and libraries used for the pre-processing and analysis of the data should be described and cited, including version numbers (item 7.9).

Reproducible research (8.0-8.5). Reproducible research practices serve as quality checks in the process of publication and further transparency and knowledge sharing, as detailed in the rubric proposed by $\mathrm{Schloss}^{38}$. Journals are increasingly implementing reproducible research standards that include the publishing of data and code, and those guidelines should be followed when possible $^{39,40}$. STORMS itemizes the accessibility of data, methods and code (items 8.0-8.5). If possible, raw data (item 8.1) and processed data (item 8.2) should be deposited into independently maintained public repositories that provide long-term availability, such those maintained by NCBI or EMBL-EBI. Repositories such as Zenodo (https://zenodo.org/) or Publisso (https://www.publisso.de/en/) can be used to provide a DOI for processed datasets. If data or code are not or cannot be made publicly available, even in a repository that provides restricted-access options, a description of how interested readers can access the data should be provided. As stated in item 8.0 , any protected information should be described, along with how such data can be accessed.

Results (9.0-10.4). The results should be reported as outlined below.
Descriptive data (9.0). Descriptive statistics about the study population should be reported (item 9.0). At a minimum, the age and sex of the study population should be described, and age and sex should be included for each participant in shared data files, but other important participant characteristics should be reported when possible, including medication use or lifestyle factors such as diet. Authors should consider reporting these data in a descriptive statistics table. Packages such as the tablel package in R software make the creation of such a table straightforward ${ }^{41}$.

Outcome data (10.0-10.4). The main outcomes of the study should be detailed, including descriptive information, findings of interest and the results of any additional analyses. Descriptive microbiome analysis (for example, dimension reduction, such as principal coordinates analysis, measures of diversity and gross taxonomic composition) should be reported for each group and each time point (item 10.0). This contextualizes the results of differential abundance analysis for readers. When differential abundance test results are reported, the magnitude and direction of differential abundance should be clearly stated (item 10.2) for each identifiable standardized taxonomic unit (item 10.1). Results from other types of analyses, such as metabolic function, functional potential, MAG assembly and RNAseq, should be described in the results as well (items 10.3 and 10.4). Additional results (for example, non-significant results or full differential abundance results) can be included in supplements and should not be excluded entirely. Although the problem has been known for decades ${ }^{39}$, journals across many fields are recognizing the issue of publication bias and therefore the issue of non-reporting of null results ${ }^{40}$. Including such results in publications will help to reduce the severity of this bias and improve future systematic reviews and meta-analyses.

Discussion (11.0-14.0). Most recommendations for the discussion section are similar to those of STROBE, including a discussion of the limitations of the present study and associated methods (item 13.0). One additional recommendation is made in the STORMS guidelines: the potential for biases and how they would influence the study findings (item 13.1) should be discussed. Many forms of bias, such as residual/unmeasured confounding, bias related to compositional analysis ${ }^{42}$, measurement bias or selection bias ${ }^{43}$, could affect 
the interpretation of the results of the study, and it is important to acknowledge potential sources of bias in discussion of the results ${ }^{44}$. As described in STROBE, authors should also consider the generalizability of their findings and if these findings could be applicable to the target population or other populations (item 13.2). If different forms of bias were not assessed or assumed to be negligible, this should be stated. Finally, authors should discuss potential future or ongoing research based on findings of the present study (item 14.0).

Other information (15.0-17.0). In addition to including a statement of funding (item 15.0), authors should also include acknowledgements and a conflicts of interest statement (items 15.1 and 15.2, respectively). Conflicts of interest statements should be written according to the criteria established by the journal. Finally, the paper should state where supplementary materials and data can be accessed (items 16.0 and 17.0).

\section{Implementation}

The STORMS checklist can streamline peer review by providing both a checklist for assessing for completeness and a roadmap with pointers to the manuscript. We recommend that before submission, authors use the 'Comments' field to provide explanations where warranted and to refer to relevant sections of the manuscript, to make the work of peer reviewers more straightforward and more accurate. We provide two examples of pre-publication use of STORMS: (i) a multi-site study of associations between essential hypertension and gut microbial metabolic pathways ${ }^{45}$; and (ii) an observational study of the stool microbiota of multiple host species ${ }^{46}$. Additional post-publication examples are also available ${ }^{47,48}$ that highlight the fact that references to line numbers must be updated during production or they will continue to refer to the pre-print version.

\section{Discussion}

The STORMS checklist for reporting on human microbiome studies was developed with the following priorities: the checklist should (1) be easy to understand and use by researchers from various fields, through straightforward use of language and pruning of items rarely relevant to the current literature; (2) be organized in the outline of a manuscript, so it can serve as a tool for authors and for peer reviewers, particularly when included in manuscript submission as a supplemental table with comments; (3) assist in the complete and organized reporting of a study, not in enforcing any particular methods; (4) reuse or modify items from related checklists where relevant; and (5) represent consensus across a broad cross-section of the human microbiome research community. The checklist facilitates manuscript authors in providing a complete, concise and organized description of their study and its findings. Included as a supplemental table to a manuscript, it also supports efficient peer review and post-publication interpretation.

Although other efforts to extend STROBE for microbiome and metagenomics studies have been proposed ${ }^{9}$ and laboratory-focused reporting checklists have been released ${ }^{4,49}$, to our knowledge, STORMS is the first comprehensive reporting checklist for human microbiome research. We aim for the STORMS guidelines to improve the quality and transparency of microbiome epidemiology studies by introducing a shared grammar of study reporting in a structured checklist format. Reporting checklists introduced in other disciplines have been shown to improve the quality of journal articles ${ }^{5,6}$.

A major strength of the STORMS checklist is the rigor and transparency of its development by a diverse, multidisciplinary consortium of subject-matter experts. The development of the STORMS checklist is an ongoing process, and new versions of the checklist will be released to reflect evolving standards and technological processes. A version-control system with a change log has been implemented, and annual reviews of the checklist are planned.
Additionally, the working group plans to evaluate the impact of the STORMS checklist on microbiome reporting by examining how many articles fulfill checklist items before and after its release. We invite interested readers to join the STORMS Consortium by contacting the corresponding author or by visiting the consortium website for more information (https://www.stormsmicrobiome.org/). We also encourage journals to include the STORMS checklist in their instructions to authors and to advise peer reviewers to consult the checklist when reviewing submissions.

There are some limitations to the STORMS checklist. The checklist was not created to assess study or methodological rigor. It is meant to aid authors' organization and ease the process of reader assessment of how studies are conducted and analyzed. Conclusions about the quality of studies should not be made on the basis of their adherence to STORMS guidelines, although we expect the reporting guidelines to help readers review studies critically. The STORMS checklist does not encourage, discourage or assume the use of null hypothesis significance testing ${ }^{50}$ or methods of compositional data analysis ${ }^{51}$, topics of some controversy in the field. In general, the checklist avoids reference to or guidance on specific statistical methodological decisions.

Through the efforts of the STORMS Consortium working in an iterative, transparent and collaborative process, the STORMS checklist provides a roadmap for researchers in reporting the results of a human microbiome study. The STORMS Consortium believes that the checklist is sufficiently flexible and user-friendly to support widespread adoption and contribution to microbiome study standards. Its adoption will ideally encourage thoughtful study design, reproducibility, collaboration and open knowledge sharing between research groups as they explore the human microbiome.

\section{Data availability}

The STORMS checklist as a versioned spreadsheet and the analytical sample size flowchart as editable PPTX and PDF files are available for adaptation and inclusion in manuscripts at https://www. stormsmicrobiome.org/.

Received: 1 December 2020; Accepted: 23 September 2021; Published online: 17 November 2021

\section{References}

1. Wirbel, J. et al. Meta-analysis of fecal metagenomes reveals global microbial signatures that are specific for colorectal cancer. Nat. Med. 25, 679-689 (2019).

2. Simoneau, J., Dumontier, S., Gosselin, R. \& Scott, M. S. Current RNA-seq methodology reporting limits reproducibility. Brief. Bioinform. 22, 140-145 (2021)

3. Ten Hoopen, P. et al. The metagenomic data life-cycle: standards and best practices. Gigascience 6, 1-11 (2017).

4. Yilmaz, P. et al. Minimum information about a marker gene sequence (MIMARKS) and minimum information about any (x) sequence (MIxS) specifications. Nat. Biotechnol. 29, 415-420 (2011).

5. Moher, D., Jones, A. \& Lepage, L. Use of the CONSORT statement and quality of reports of randomized trials: a comparative before-and-after evaluation. J. Am. Med. Assoc. 285, 1992-1995 (2001).

6. Plint, A. C. et al. Does the CONSORT checklist improve the quality of reports of randomised controlled trials? Syst. Rev. 185, 5 (2006).

7. Vandenbroucke, J. P. et al. Strengthening the reporting of observational studies in epidemiology (STROBE): explanation and elaboration. PLoS Med. 4, e297 (2007)

8. Little, J. et al. STrengthening the REporting of Genetic Association Studies (STREGA) - an extension of the STROBE statement. Genet. Epidemiol. 33, 581-598 (2009)

9. Bharucha, T. et al. STROBE-metagenomics: a STROBE extension statement to guide the reporting of metagenomics studies. Lancet Infect. Dis. 20, e251-e260 (2020).

10. Sinha, R. et al. Assessment of variation in microbial community amplicon sequencing by the Microbiome Quality Control (MBQC) project consortium. Nat. Biotechnol. 35, 1077-1086 (2017).

11. Costea, P. I. et al. Towards standards for human fecal sample processing in metagenomic studies. Nat. Biotechnol. 35, 1069-1076 (2017). 
12. Santiago, A. et al. Processing faecal samples: a step forward for standards in microbial community analysis. BMC Microbiol. 14, 112 (2014).

13. Turner, P. et al. Microbiology Investigation Criteria for Reporting Objectively (MICRO): a framework for the reporting and interpretation of clinical microbiology data. BMC Med. 17, 70 (2019).

14. Kerns, S. L. et al. STROGAR - STrengthening the Reporting Of Genetic Association studies in Radiogenomics - ScienceDirect. Radiother. Oncol. 110, 182-188 (2014).

15. Sinha, R., Abnet, C. C., White, O., Knight, R. \& Huttenhower, C. The microbiome quality control project: baseline study design and future directions. Genome Biol. 16, 276 (2015).

16. Badal, V. D. et al. Challenges in the construction of knowledge bases for human microbiome-disease associations. Microbiome 7, 129 (2019).

17. von Elm, E. et al. The Strengthening the Reporting of Observational Studies in Epidemiology (STROBE) statement: guidelines for reporting observational studies. J. Clin. Epidemiol. 61, 344-349 (2008).

18. Gallo, V. et al. STrengthening the Reporting of OBservational studies in Epidemiology - Molecular Epidemiology (STROBE-ME): an extension of the STROBE statement. Eur. J. Clin. Invest. 42, 1-16 (2012).

19. Kerns, S. L. et al. STROGAR - STrengthening the Reporting Of Genetic Association studies in Radiogenomics. Radiother. Oncol. 110, 182-188 (2014).

20. Sinha, R. et al. Next steps in studying the human microbiome and health in prospective studies, Bethesda, MD, May 16-17, 2017. Microbiome 6, 210 (2018).

21. Hornung, B. V. H., Zwittink, R. D. \& Kuijper, E. J. Issues and current standards of controls in microbiome research. FEMS Microbiol. Ecol. 95 fiz045 (2019).

22. Min, Y. et al. Sex-specific association between gut microbiome and fat distribution. Nat. Commun. 10, 2408 (2019).

23. Pearce, N. Classification of epidemiological study designs. Int. J. Epidemiol. 41, 393-397 (2012).

24. Jin, Y., Wu, S., Zeng, Z. \& Fu, Z. Effects of environmental pollutants on gut microbiota. Environ. Pollut. 222, 1-9 (2017).

25. Chen, L., Zhang, Y.-H., Huang, T. \& Cai, Y.-D. Gene expression profiling gut microbiota in different races of humans. Sci. Rep. 6, 23075 (2016).

26. Yatsunenko, T. et al. Human gut microbiome viewed across age and geography. Nature 486, 222-227 (2012).

27. Patino, C. M., Ferreira, J. C., Patino, C. M. \& Ferreira, J. C. Inclusion and exclusion criteria in research studies: definitions and why they matter. J. Bras. Pneumol. 44, 84-84 (2018).

28. Yan, L. et al. OSAT: a tool for sample-to-batch allocations in genomics experiments. BMC Genomics 13, 689 (2012).

29. VanderWeele, T. J. Principles of confounder selection. Eur. J. Epidemiol. 34, 211-219 (2019).

30. Hernán, M. A., Hernández-Díaz, S., Werler, M. M. \& Mitchell, A. A. Causal knowledge as a prerequisite for confounding evaluation: an application to birth defects epidemiology. Am. J. Epidemiol. 155, 176-184 (2002).

31. Lv, B.-M., Quan, Y. \& Zhang, H.-Y. Causal inference in microbiome medicine: principles and applications. Trends Microbiol. 29, 736-746 (2021).

32. Ghosh, T. S., Das, M., Jeffery, I. B. \& O’Toole, P. W. Adjusting for age improves identification of gut microbiome alterations in multiple diseases. eLife 9, e50240 (2020).

33. Soneson, C., Gerster, S. \& Delorenzi, M. Batch effect confounding leads to strong bias in performance estimates obtained by cross-validation. PLoS One 9, e100335 (2014).

34. Cole, S. R. et al. Illustrating bias due to conditioning on a collider. Int. J. Epidemiol. 39, 417-420 (2010).

35. Textor, J., van der Zander, B., Gilthorpe, M. S., Liśkiewicz, M. \& Ellison, G. T. $\mathrm{H}$. Robust causal inference using directed acyclic graphs: the R package 'dagitty'. Int. J. Epidemiol. 45, 1887-1894 (2016).

36. Loughman, A. et al. Gut microbiota composition during infancy and subsequent behavioural outcomes. EBioMedicine 52, 102640 (2020).

37. Schooling, C. M. Selection bias in population-representative studies? A commentary on Deaton and Cartwright. Soc. Sci. Med. 210, 70 (2018).

38. Schloss, P. D. Identifying and overcoming threats to reproducibility, replicability, robustness, and generalizability in microbiome research. $m$ Bio 9, e00525-18 (2018).

39. Rosenthal, R. The file drawer problem and tolerance for null results. Psychol. Bull. 86, 638-641 (1979).

40. Munafò, M. \& Neill, J. Null is beautiful: On the importance of publishing null results. J. Psychopharmacol. 30, 585-585 (2016).

41. Rich, B. Tables of Descriptive Statistics in HTML [R package table1 version 1.4.2] (2021)

42. McLaren, M. R., Willis, A. D. \& Callahan, B. J. Consistent and correctable bias in metagenomic sequencing experiments. eLife 8, e46923 (2019).
43. Hernán, M. \& Robins, J.M. Causal Inference: What If (Chapman \& Hall/CRC, 2020).

44. Höfler, M. et al. Writing a discussion section: how to integrate substantive and statistical expertise. BMC Med. Res. Methodol. 18, 34 (2018).

45. Nakai, M. et al. Essential hypertension is associated with changes in gut microbial metabolic pathways: a multisite analysis of ambulatory blood pressure. Hypertension 78, 804-815 (2021).

46. Thingholm, L. B. et al. Ecology impacts the decrease of Spirochaetes and Prevotella in the fecal gut microbiota of urban humans. BMC Microbiol 21, 276 (2021).

47. Wang, Q. et al. Linking gut microbiome to bone mineral density: a shotgun metagenomic dataset from 361 elderly women. Gigabyte 2021, 1-7 (2021).

48. Chen, C. et al. The female urinary microbiota in relation to the reproductive tract microbiota. Gigabyte 2020, 1-9 (2020).

49. Raes, J., Foerstner, K. U. \& Bork, P. Get the most out of your metagenome: computational analysis of environmental sequence data. Curr. Opin. Microbiol. 10, 490-498 (2007).

50. Szucs, D. \& Ioannidis, J. P. A. When null hypothesis significance testing is unsuitable for research: a reassessment. Front. Hum. Neurosci. 11, 390 (2017).

51. Gloor, G. B., Macklaim, J. M., Pawlowsky-Glahn, V. \& Egozcue, J. J. Microbiome datasets are compositional: and this is not optional. Front. Microbiol. 8, 2224 (2017).

\section{Acknowledgements}

Research reported in this publication was supported by the National Cancer Institute of the National Institutes of Health under award number 5R01CA230551. The content is solely the responsibility of the authors and does not necessarily represent the official views of the National Institutes of Health. Additionally, J.B.D. was supported by the Leverhulme Trust.

\section{Author contributions}

The Genomic Standards Consortium and Massive Analysis and Quality Control Society provided feedback and approved the final checklist; C.M., A.R., J.B.D., H.E.J. and L.W. conceived of the checklist; C.M., A.R., J.v.d.W., N.S., C.H., J.B.D., H.E.J. and L.W. developed the initial checklist; C.M., F.Z., S.E., R.A., J.B.D., H.E.J. and L.W. tested the checklist; C.M. and L.W. drafted the manuscript; F.Z.M. created manuscript headings and analytical sample size flowchart figures; C.M. created supplemental tables; A.R., F.Z. S.E., L.G., L.J.K., K.E., J.v.d.W., A.L., F.Z.M., D.A.M., M.A., R.A., F. Beghini, K.B., A.B., J. Bisanz, H.C.B., G.A.B., F. Bushman, D.C., G.C., M.C.C., P.D.C., J.F.C., R.T.D., S.D., E.E., J.F., R.D.F., A.A.F., S.F., A.F., C.F., J.G., E.G., B.H.-K., S. Handley, P.H., S. Holmes, J.P.J., L.K., R.K., D. Knights, O.K., D. Kown, M.L., B.L., D.P.B.M., A.C.M., S.M., N.T.M., L.N., M.O., N.P., E.P., J.R., M.R.R., J.S.E., M.R., R.B.S., P.D.S., L.S., E. Segal, M.S., T.S., E. Smirnova, H.S., J.L.S., S.S., L.B.T., P.J.T., V.U., R.L.W., P.W., G.Z., M.Z., N.Z., N.S., J.B.D., H.E.J. and C.H. provided feedback on the checklist and manuscript; and all authors reviewed and approved the manuscript.

\section{Competing interests}

J.S.E. is employed by a research center that belongs to a food company (Grupo Empresarial Nutresa). T.J.S. is a shareholder for Resilient Biotics. H.S. received unrestricted study grants from Biocodex; received board membership, consultancy or lecture fees from Carenity, Abbvie, Astellas, Danone, Ferring, Mayoly Spindler, Merck Sharp \& Dohme, Novartis, Roche, Tillots, Enterome, Maat, BiomX, Biose, Novartis and Takeda; and is a co-founder of Exeliom Biosciences. L.B.T. is an employee and shareholder of BiomCare. P.W. is listed as an inventor on patents PCT/EP2012/065178 and PCT/EP2013/052134, and is a member of the scientific steering committee for a clinical trial by 4D Pharma. T.Y. is a founder and board member of Metabologenomics and Metagen Therapeutics. L.Z. is a co-founder of Notitia Biotechnologies.

\section{Additional information}

Supplementary information The online version contains supplementary material available at https://doi.org/10.1038/s41591-021-01552-x.

Correspondence and requests for materials should be addressed to Levi Waldron.

Peer review information Nature Medicine thanks the anonymous reviewers for their contribution to the peer review of this work. Joao Monteiro was the primary editor on this article and managed its editorial process and peer review in collaboration with the rest of the editorial team.

Reprints and permissions information is available at www.nature.com/reprints.

Publisher's note Springer Nature remains neutral with regard to jurisdictional claims in published maps and institutional affiliations.

(c) The Author(s), under exclusive licence to Springer Nature America, Inc. 2021 
${ }^{1}$ CUNY Graduate School of Public Health and Health Policy, Institute for Implementation Science in Public Health, New York, NY, USA. ${ }^{2}$ University of North Carolina at Chapel Hill, Chapel Hill, NC, USA. ${ }^{3}$ Julius Center for Health Sciences and Primary Care, University Medical Center Utrecht, Utrecht University, Utrecht, the Netherlands. ${ }^{4}$ Food \& Mood Centre, The Institute for Mental and Physical Health and Clinical Translation, Deakin University, Geelong, Victoria, Australia. ${ }^{5}$ Hypertension Research Laboratory, School of Biological Sciences, Faculty of Science, Monash University, Melbourne, Victoria, Australia. ${ }^{6}$ Department of Metabolism, Digestion and Reproduction, Faculty of Medicine, Imperial College London, London, UK. ${ }^{7}$ Novo Nordisk Foundation Center for Basic Metabolic Research, Faculty of Health and Medical Sciences, University of Copenhagen, Copenhagen, Denmark. ${ }^{8}$ Department CIBIO, University of Trento, Trento, Italy. ${ }^{9}$ Department of Biology, University of British Columbia-Okanagan Campus, Kelowna, British Columbia, Canada. ${ }^{10}$ Division of Hematology and Division of Bone Marrow Transplantation, Department of Medicine, and Department of Genetics, Stanford University, Stanford, CA, USA. "Department of Biochemistry and Molecular Biology, Pennsylvania State University, University Park, PA, USA. ${ }^{2}$ Division of Gastroenterology and Hepatology, Department of Medicine, Cedars-Sinai Medical Center, Los Angeles, CA, USA. ${ }^{13}$ Genentech, South San Francisco, CA, USA. ${ }^{14}$ Center for Microbiome Engineering and Data Analysis, Department of Microbiology and Immunology, Virginia Commonwealth University, Richmond, VA, USA. ${ }^{15}$ University of Pennsylvania, Philadelphia, PA, USA. ${ }^{16} \mathrm{~F}$. Widjaja Foundation Inflammatory Bowel and Immunobiology Research Institute, Cedars-Sinai Medical Center, Los Angeles, CA, USA. ${ }^{17}$ Department of Psychiatry and Neurobehavioural Science, and APC Microbiome Ireland, University College Cork, Cork, Ireland. ${ }^{18}$ Institute of Agrochemistry and Food Technology-National Research Council, Valencia, Spain. ${ }^{19}$ Teagasc Food Research Centre-Moorepark, Cork, Ireland. ${ }^{20} \mathrm{APC}$ Microbiome Ireland, University College Cork, Cork, Ireland. ${ }^{21}$ VistaMilk, Cork, Ireland. ${ }^{22}$ Department of Anatomy and Neuroscience, University College Cork, Cork, Ireland. ${ }^{23}$ School of Public Health, University of Minnesota, Minneapolis, MN, USA. ${ }^{24}$ Immunology Department, Weizmann Institute of Science, Rehovot, Israel. ${ }^{25}$ Microbiome and Cancer Division, Deutsches Krebsforschungszentrum, Heidelberg, Germany. ${ }^{26}$ Vidarium-Nutrition, Health and Wellness Research Center, Grupo Empresarial Nutresa, Medellin, Colombia. ${ }^{27}$ European Molecular Biology Laboratory, European Bioinformatics Institute, Wellcome Genome Campus, Hinxton, UK. ${ }^{28}$ Department of Bioinformatics and Genomics, University of North Carolina at Charlotte, Charlotte, NC, USA. ${ }^{29}$ Experimental and Clinical Research Center, Max Delbrück Center for Molecular Medicine and Charité University Hospital, Berlin, Germany. ${ }^{30}$ Institute of Clinical Molecular Biology, Kiel University, Kiel, Germany. ${ }^{31} \mathrm{HK} 3$ Lab, Rovereto, Italy. ${ }^{32}$ Department of Pediatrics and Scripps Institution of Oceanography, University of California San Diego, La Jolla, CA, USA. ${ }^{33}$ Department of Dermatology, Perelman School of Medicine, University of Pennsylvania, Philadelphia, PA, USA. ${ }^{34}$ Princess Margaret Cancer Centre, University Health Network, Toronto, Ontario, Canada. ${ }^{35}$ Department of Pathology and Immunology, Washington University School of Medicine, St. Louis, MO, USA. ${ }^{36}$ McCourt School of Public Policy, Georgetown University, Washington, DC, USA. ${ }^{37}$ Department of Statistics, Stanford University, Stanford, CA, USA. ${ }^{38}$ Division of Digestive Diseases, David Geffen School of Medicine at UCLA, Los Angeles, CA, USA. ${ }^{39}$ Department of Medical Informatics and Clinical Epidemiology, Oregon Health \& Science University, Portland, OR, USA. ${ }^{40} \mathrm{Center}$ for Microbiome Innovation, University of California San Diego, La Jolla, CA, USA. ${ }^{41}$ Department of Computer Science and Engineering, University of Minnesota, Minneapolis, MN, USA. ${ }^{42}$ Biotechnology Institute, University of Minnesota, Saint Paul, MN, USA. ${ }^{43}$ Azrieli Faculty of Medicine, Bar Ilan University, Safed, Israel. ${ }^{44}$ Division of Infectious Diseases, Massachusetts General Hospital, Boston, MA, USA. ${ }^{45}$ Department of Pharmacology, Dalhousie University, Halifax, Nova Scotia, Canada. ${ }^{46}$ University of Maryland School of Medicine, Institute of Human Virology, Baltimore, MD, USA. ${ }^{47}$ Department of Computational Biology of Infection Research, Helmholtz Centre for Infection Research, Brunswick, Germany. ${ }^{48} \mathrm{OHSU}$ Knight Cancer Institute, Portland, OR, USA. ${ }^{49}$ Department of Epidemiology, Johns Hopkins Bloomberg School of Public Health, Baltimore, MD, USA. ${ }^{50}$ Department of Experimental Oncology, Istituto di Ricovero e Cura a Carattere Scientifico-Istituto Europeo di Oncologia, Milan, Italy. ${ }^{51}$ Department of Microbiology and Immunology, Stanford University, Stanford, CA, USA. ${ }^{52}$ Department of Immunobiology, Yale University School of Medicine, New Haven, CT, USA. ${ }^{53}$ Department of Agricultural Sciences, University of Naples Federico II, Portici, Italy. ${ }^{54}$ Department of Microbiology and Immunology, Rega institute, KU Leuven and VIB Center for Microbiology, Leuven, Belgium. ${ }^{55}$ Department of Chemistry, University of North Carolina at Chapel Hill, Chapel Hill, NC, USA. ${ }^{56}$ Institute of Clinical Molecular Biology, Christian Albrechts University of Kiel, Kiel, Germany. ${ }^{57}$ Center for Gastrointestinal Biology and Disease, Division of Gastroenterology and Hepatology, Department of Medicine, University of North Carolina at Chapel Hill, Chapel Hill, NC, USA. ${ }^{58}$ Department of Microbiology \& Immunology, University of Michigan, Ann Arbor, MI, USA. ${ }^{5}$ University of Maryland School of Medicine, Institute for Genome Sciences, Baltimore, MD, USA. ${ }^{60}$ Department of Computer Science, Weizmann Institute of Science, Rehovot, Israel. ${ }^{61}$ Department of Microbiology and Department of Statistics, Oregon State University, Corvallis, OR, USA. ${ }^{62}$ Department of Biostatistics, Virginia Commonwealth University, Richmond, VA, USA. ${ }^{33}$ Gastroenterology Department, Centre de Recherche Saint-Antoine, INSERM, Assistance Publique-Hôpitaux de Paris, Saint Antoine Hospital, Sorbonne Université, Paris, France. ${ }^{64}$ Vaccine and Infectious Disease Division, Fred Hutchinson Cancer Research Center, Seattle, WA, USA. ${ }^{65}$ Department of Microbiology and Immunology, University of California San Francisco, San Francisco, CA, USA. ${ }^{66}$ Critical Path Institute, Tucson, AZ, USA. ${ }^{67}$ Luxembourg Centre for Systems Biomedicine, University of Luxembourg, Esch-sur-Alzette, Luxembourg. ${ }^{68}$ Department of Life Sciences and Medicine, Faculty of Science, Technology and Medicine, University of Luxembourg, Esch-sur-Alzette, Luxembourg. ${ }^{69}$ Department of Life Science and Technology, Tokyo Institute of Technology, Tokyo, Japan. ${ }^{70}$ Structural and Computational Biology Unit, European Molecular Biology Laboratory, Heidelberg, Germany. ${ }^{71}$ Department of Biostatistics, Johns Hopkins Bloomberg School of Public Health, Baltimore, MD, USA. ${ }^{72}$ Department of Biochemistry and Microbiology, School of Environmental and Biological Sciences, Rutgers University, New Brunswick, NJ, USA. ${ }^{73}$ JMP Life Sciences, SAS Institute, Cary, NC, USA. ${ }^{74}$ Department of Data Sciences, Dana-Farber Cancer Institute, Boston, MA, USA. ${ }^{75}$ Eisai, Woodcliff Lake, NJ, USA. ${ }^{76} \mathrm{Clinical}$ Bioinformatics Area, Hospital Virgen del Rocio, Sevilla, Spain. ${ }^{77} \mathrm{College}$ of Pharmaceutical Sciences, Zhejiang University, Hangzhou, China. ${ }^{78}$ Experimental Pediatric Oncology, University Children's Hospital, Cologne, Germany. ${ }^{79}$ Center for Molecular Medicine Cologne, Medical Faculty, University of Cologne, Cologne, Germany. ${ }^{80}$ Q2 Solutions-EA Genomics, Durham, NC, USA. ${ }^{81}$ Immuneering, Cambridge, MA, USA. ${ }^{82}$ Weill Cornell Medicine, New York, NY, USA. ${ }^{83}$ Australian Institute for Bioengineering and Nanotechnology, University of Queensland, Brisbane, Queensland, Australia. ${ }^{84}$ Oxford e-Research Centre, Department of Engineering Science, University of Oxford, Oxford, UK. ${ }^{85}$ Institute for Molecular Medicine Finland, University of Helsinki, Helsinki, Finland. ${ }^{86}$ State Key Laboratory of Genetic Engineering, Human Phenome Institute, School of Life Sciences and Shanghai Cancer Center, Fudan University, Shanghai, China. ${ }^{87}$ Office of Computational Science, Office of Translational Sciences, Center for Drug Evaluation and Research, Washington, DC, USA. ${ }^{88}$ Division of Bioinformatics and Biostatistics, National Center for Toxicological Research, Food \& Drug Administration, Jefferson, AR, USA. ${ }^{99}$ Scientific Discovery and Genomics, SAS Institute, Cary, NC, USA. ${ }^{90} \mathrm{GigaScience} \mathrm{Database,}$ BGI Hong Kong, Hong Kong, China. ${ }^{91}$ Harvard T. H. Chan School of Public Health, Boston, MA, USA. ${ }^{92}$ Department of Sociology, Leverhulme Centre for Demographic Science, University of Oxford, Oxford, UK. ${ }^{93}$ These authors contributed equally: Curtis Huttenhower, Jennifer B. Dowd, Heidi E. Jones, Levi

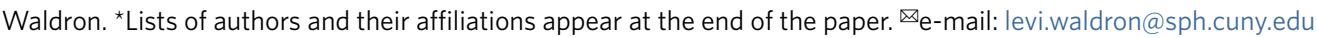


Genomic Standards Consortium

Robert D. Finn ${ }^{27}$, Lynn Schriml ${ }^{59}$, Ramona L. Walls ${ }^{66}$, Christopher Hunter ${ }^{90}$ and Susanna-Assunta Sansone ${ }^{84}$

Massive Analysis and Quality Control Society

Cesare Furlanello ${ }^{31}$, Benjamin Haibe-Kains ${ }^{34}$, Wenjun Bao ${ }^{73}$, Aedin Culhane ${ }^{74}$,

Viswanath Devanarayan ${ }^{75}$, Joaquin Dopazo ${ }^{76}$, Xiaohui Fan ${ }^{77}$, Matthias Fischer ${ }^{78,79}$, Wendell Jones ${ }^{80}$, Rebecca Kusko ${ }^{81}$, Christopher E. Mason ${ }^{82}$, Tim R. Mercer ${ }^{83}$, Andreas Scherer ${ }^{85}$, Leming Shi ${ }^{86}$, Shraddha Thakkar ${ }^{87}$, Weida Tong ${ }^{88}$, Russ Wolfinger ${ }^{89}$ and Susanna-Assunta Sansone ${ }^{84}$ 